\section{Software for the generation and display of random-dot cinematograms on the Macintosh computer}

\author{
SCOTT B. STEINMAN \\ University of Missouri, St. Louis, Missouri \\ and \\ MARK NAWROT \\ Vanderbilt University, Nashville, Tennessee
}

The software package DotMovie 1.3.8 was designed to create and display dynamic random-dot cinematograms for studies of motion perception on Macintosh II microcomputers. In each frame of the cinematogram, some dots move in random uncorrelated directions, while other dots move with constant speed in one direction. As the relative number of dots moving in a correlated direction increases, the observer sees flow in the direction of the movement. The software described facilitates the creation of random-dot cinematograms, their presentation, and collection of data from subjects' responses.

The software package described here, DotMovie 1.3.8, was designed to create and display dynamic random-dot cinematograms on a Macintosh II series computer in studies of motion perception. In each frame of the cinematogram, some dots (noise dots) move in random, uncorrelated, directions. The remaining correlated dots move with constant speed in one direction; this movement is also referred to as the motion signal. From one cinematogram frame to the next, there is random variation in which a subset of the dots will move in the signal direction. As a result, signal and random motions are spatially intermingled. When sufficient dots move in a correlated fashion, thereby creating a large enough signal, the observer sees a global flow in the direction of the correlated movements (Williams \& Sekuler, 1984). By varying either the proportion of correlated signal dots to randomly moving noise dots or the relative contrasts of the signal and noise dots (van Doorn \& Koenderink, 1983), one can measure motion thresholds.

The DotMovie program coordinates the creation of random-dot cinematograms, their subsequent presentation to an experimental subject, and the collection of the subject's responses through the keyboard or a remote keypad device. The responses are saved to disk file for later analysis. The program is entirely menu and mouse driven and conforms to Macintosh user interface standards. The program was written with Think $\mathrm{C}$, Version 5.0.2

Correspondence should be addressed to S. B. Steinman, University of Missouri, School of Optometry, 8001 Natural Bridge Road, St. Louis, MO 63121
(Symantec Corporation, Cupertino, CA) and the ResEdit resource editor, Version 2.1.1 (Apple Computer, Cupertino, CA). DotMovie is compatible with the Macintosh System 7.0 operating system.

The DotMovie software allows the experimenter to easily build experiments dealing with random-dot motion. Either gray-scale or color dots may be presented, permitting the manipulation of dot contrast or the study of color and motion interactions. Cinematograms are composed of up to 100 animation frames, each containing up to 200 dots displayed in a circular aperture on the Macintosh's screen. A description of the cinematogram sequencethe number of dots presented, their gray-scale luminances or colors, their velocity, and so forth-is stored as a single disk file. These cinematogram parameter files are given distinct identifying icons for easy identification. When an experiment is conducted, a parameter file is read from disk and a series of related cinematograms are presented to the subject.

\section{Cinematogram Creation Module}

The dot cinematogram program consists of two parts. The first part allows the experimenter to build a description of a random-dot cinematogram and store it to disk. For a given experiment, an independent variable, such as dot displacement, dot velocity, motion signal strength, or motion range is set. The experimenter may then select up to nine levels or values of the independent variable to be presented in random order on each trial of the experiment. A series of dialog boxes prompts the experimenter to set other relevant motion parameters such as the total number of dots in the cinematograms, the percentage of dots that will move together as the signal (correlated dots), the dot motion direction, the dot displacement per frame, the number of frames in the cinematogram, the frame timing, the size of the dot field and of the individual dots, the contrast of the signal and noise dots, the color of the dots, the range of directions in the noise dots, and so forth. These variables will affect the speed of dot motion, the exposure duration of the cinematogram, and the smoothness of motion. Individual frames may be presented for a period as short as $15 \mathrm{msec}$, permitting rapid velocities to be tested. In addition, the testing distance and the eccentricity of the dot field from a central fixation point may be selected.

The experimenter also has the option of selecting several experimental paradigms. Motion detection thresholds may be measured by varying the percentage of signal dots, thereby varying the strength of the motion signal (Williams \& Sekuler, 1984). Alternatively, the signal strength may be varied by changing the relative contrasts of the signal and noise dots (van Doorn \& Koenderink, 1983). The experimenter also has the option of selecting the degree of randomness in the uncorrelated (noise) dots by restricting the range of directions in which they move. 
Direction discrimination thresholds may be assessed in a four-alternative forced-choice paradigm as the direction of the correlated signal dot motion is varied. Finally, velocity discrimination thresholds may be collected with the method of single stimuli as the dot displacement per frame and frame timing are varied.

The DotMovie program allows the experimenter to preview a cinematogram before saving its description to disk. In addition, a menu selection presents a special dialog box containing the cinematogram motion parameters in real-world units (minutes of arc, degrees per second, milliseconds) rather than in units of pixels or screen refreshes.

\section{Experimental Execution Module}

The second section of the program presents cinematograms to the observer and collects responses. When a parameter file is read prior to the experiment's being run, the proper graphics environment for presenting the cinematogram defined by those parameters is restored. This allows successive cinematograms shown to the subject during an experiment to have vastly different motion parameters, such as different dot contrasts or velocities, and still be displayed accurately. The precision of the motion velocity is ensured by the use of Slot Manager and Vertical Retrace Manager timing routines.

On each trial, the level of the stimulus independent variable (e.g., displacement, velocity, contrast, or signal strength) is randomly selected from up to nine values stored in the parameter file, permitting the experimenter to use a yes/no or forced-choice method of constant stimuli paradigm for data collection. The subject's responses are collected through the keyboard and saved to a disk file. Alternatively, remote keypads available for the Macintosh computer may be used as a keyboard replacement for collecting responses. The experimenter may select via the menu to provide auditory feedback of the response correctness to the observer.

The output data files contain textual information about the subject, the experimental paradigm, and the motion stimulus settings. In addition, there is a list of each stimulus level presented to the observer, along with the observer's response and the correctness of the response for each trial of the experiment. Summary statistics are listed for each independent variable level and for each motion direction.

The data file also contains a custom Macintosh resource containing each independent variable level and the number of trials and number of correct responses at each level. This resource is read directly by a psychometric function fitting program in our laboratory. A ResEdit resource template for this data file is provided for users who wish to write their own psychometric function analysis program. Similarly, the experimental settings are saved as resources in cinematogram parameter files. This allows the experimenter to use the ResEdit resource editor to examine the contents of the parameter file to view or change the parameters used to present a given class of cinematograms. A ResEdit template is provided for examining and modifying the experimental parameter resource.

If the observer is threatened by the use of computers, the experimenter has options available for simplifying the subject's task. If the computer system has two monitors, both may be used simultaneously-one for stimulus display to the observer and the other for the user interface. This reduces the possibility of distracting the observer with dialog boxes and menus. The menu choices that may be selected by the observer are limited solely to those that start an experimental session. The menus are therefore less complicated, allowing observers to run the program with a minimum of assistance from the experimenter. The disabling of cinematogram creation options from the menus also prevents observers from accidentally modifying motion parameters.

\section{System Requirements}

The program requires a Macintosh II series machine to make use of 32-bit QuickDraw, the Palette Manager, and Vertical Retrace Manager timing routines available only on the Mac II series machines (Steinman \& Nawrot, 1992). At least $2.5 \mathrm{MB}$ of free memory is suggested to create and display cinematograms. This amount will increase if larger cinematograms are used. The timing routines assume that the refresh rate of the screen is $67 \mathrm{~Hz}$, as on the AppleColor RGB monitor. The program has been tested on the Macintosh IIfx, IIci, IIsi, IIx, and II computers, with and without the benefit of the $8 \cdot 24 \mathrm{GC}$ graphics accelerator board. The graphics accelerator board permits the use of larger areas of dots in the cinematograms.

\section{Availability}

The compiled program is available from S. B. Steinman (please send a self-addressed stamped disk mailer and diskette). Some of the programming issues involved in writing the program are explained by Steinman and Nawrot (1992) and Steinman (in press).

\section{REFERENCES}

Steinman, S. B. (in press). Simple frame animation for the Macintosh computer. MacTutor: The Macintosh Programming Journal.

Steinman, S. B., \& Nawrot, M. (1992). Real-time color frame animation for visual psychophysics on the Macintosh computer. Behavior Research Methods, Instruments, \& Computers, 24, 439-452.

Van Doorn, A. J., \& Koenderink, J. J. (1983). Detectability of velocity gradients in moving random-dot patterns. Vision Research, 23, 799-804.

Wiluams, D. W., Sekuler, R. (1984). Coherent global motion percepts from stochastic local motions. Vision Research, 24, 55-62.

(Manuscript received July 8, 1991; revision accepted for publication April 8, 1992.) 